\title{
The impact of experimental manipulation on neutrophil immunophenotyping
}

\author{
Gerasimina Tsinti, Anastasios E Germenis and Matthaios Speletas* \\ Department of Immunology and Histocompatibility, Faculty of Medicine, School of Health Sciences, University of Thessaly, Larissa, Greece
}

\begin{abstract}
The interest of developing functional and other experimental procedures on polymorphonuclear neutrophils (PMN) for the study of inflammatory disorders and primary immunodeficiencies is constantly increased. However, these experimental manipulations may induce significant changes on neutrophils' immunophenotyping and function. For this purpose, we investigated the effect of different experimental procedures (such as resting state, isolation and incubation with and without inflammatory stimuli) on the immunophenotype of PMN of healthy individuals, evaluating the expression pattern of specific granulocytic markers, important for chemotaxis, adhesion, opsonization, migration and phagocytosis. Our results indicate that the effect of experimental manipulation on PMN should always be taken into consideration in order to avoid misinterpreting the experimental findings.
\end{abstract}

\section{Introduction}

Polymorphonuclear neutrophils (PMN) are major effector cells in innate host defense against invading microorganisms. They migrate towards the site of infection and inflammation in order to confront the pathogens. PMN recognize pathogens through various receptors, such as scavenger, complement and immunoglobulin receptors, eliminating them either through mechanisms that involve degranulation, phagocytosis and oxidative killing into the phagosomes, or through the formation of neutrophil extracellular traps (NETs) [1,2].

During recent years there is an increasing interest in the development of procedures, where human PMN are being studied in the context of inflammatory disorders and primary immunodeficiencies $[3,4]$. In these procedures, the expression of cell surface molecules, such as adhesion molecules, immunoglobulin and complement receptors, is investigated along with functional responses to inflammatory stimuli [3-5]. In order to safely draw conclusions when investigating the role of PMN in health and disease, the in vitro data should reflect the in vivo behavior. A few previous studies have shown that specific experimental manipulation of PMN might induce changes in the expression pattern of several surface antigens [6-8].

Therefore, the aim of our study was to investigate more precisely the impact of different experimental procedures, such as isolation and incubation with and without inflammatory stimuli, on the immunophenotype of PMN of healthy individuals, evaluating the expression pattern of specific granulocytic markers, important for chemotaxis, adhesion, opsonization, migration and phagocytosis.

\section{Material and methods}

\section{Subjects}

Eight milliliters of peripheral blood were collected using heparinized syringe from 14 healthy individuals (male/female: 4/10, mean age: 41.5 years, range: $21-65$ ) with no history of autoimmunity, chronic inflammatory disorder, or malignancy. All individuals were receiving no medication during blood collection, except from two subjects under common anti-hypertensive treatment. The absence of infection/inflammation was also verified after clinical examination and the measurement of C-reactive protein (CRP) serum levels, using commercially available immunonephelometric assays (Immulite-2000, Siemens Medical Solutions, Llanberis, Gwynedd, UK), according to the manufacturer's instructions. All subjects provided written informed consent. The study was conducted in accordance with the principles of the Helsinki Declaration and was approved by the Institutional Review Board of the University Hospital of Larissa, Greece.

\section{Flow cytometry analysis}

Immunophenotyping was performed by flow cytometry on Coulter FC-500 instrument (Epics XL-MCL, 4 color analysis, Beckman-Coulter/ BC, Hialeah, FL, USA) using a multistaining protocol and commercially available reagents. Mouse anti-human immunoglobulin G (IgG) monoclonal antibodies were used for the detection of CD10 (clone: ALB1), CD11b (clone: Bear1), CD14 (clone: RMO52), CD16 (clone: 3G8), CD18 (clone: 7E4), CD64 (clone: 22), and CD66b (clone: 80H3). All the above antibodies were purchased by $\mathrm{BC}$ and were conjugated with the appropriate fluorochrome (fluorescein isothiocyanate, FITC; phycoerythrin, PE; PE- cyanine5 PE-Cy5). The percentage of fluorescent cells and the mean fluorescence intensity (MFI) were determined in each case corrected for background fluorescence, using FITC, PE, and PE-Cy5-labelled control antibodies. All lysis and staining procedures were performed at room temperature. The data analyzed were the percentile (\%) expression of each marker and the intensity of expression determined by the equation: (\%) expression $\times$ MFI / 100 . In particular, we evaluated the expression pattern of the aforementioned markers on PMN in four different conditions: (1) resting (basal) state,

*Correspondence to: Matthaios Speletas, Department of Immunology and Histocompatibility, Faculty of Medicine, School of Health Sciences, University of Thessaly, Larissa, Greece, Tel: +302410685687; E-mail: maspel@med.uth.gr

Key words: polymorphonuclear neutrophil, immunophenotyping, isolation, LPS

Received: July 05, 2018; Accepted: July 19, 2018; Published: July 23, 2018 
where PMN had not undergone any treatment, (2) normal density PMN, where neutrophil phenotype reflects changes due to the isolation procedure, (3) culture-control PMN, where neutrophil phenotype reflects changes under culture conditions without any trigger, and (4) culture-lipopolysaccharide (LPS) PMN, where neutrophil phenotype reflects changes under LPS trigger. Experimental manipulation of specimens was always begun into two hours after blood collection.

Therefore, the heparinized whole-blood samples were separated in three parts. The first part (approximately $2 \mathrm{~mL}$ ) was immediately used for immunophenotyping of PMN at resting (basal) state. The second part $(4 \mathrm{~mL})$ was used for PMN isolation and the third part (approx. 0.5$1 \mathrm{~mL}$ ) was used in culture assays, as described below:

In resting (basal) state, the erythrocytes were initially removed using $\mathrm{NH}_{4} \mathrm{Cl} / \mathrm{KHCO}_{3}$ lysis solution, and white blood cells (WBC) were washed twice with $1 \times$ PBS. After resuspension, WBC were stained with the specific monoclonal antibodies (CD10, CD11b, CD14, CD16, CD18, CD64, and CD66b) for 15 minutes. After being washed with PBS, WBCs were re-suspended in PBS and a flow cytometric evaluation in basal state was performed. Approximately 10,000 PMN were acquired per sample and cells were initially gated according to forward and side scatter characteristics.

PMN isolation was performed using the double ficoll-gradient density centrifugation. More specifically $3 \mathrm{~mL}$ of Histopaque 1119 (Sigma-Aldrich, St. Louis, Missouri, USA) were layered on top of $3 \mathrm{~mL}$ Histopaque $1077 \mathrm{~g} / \mathrm{ml}$ (Sigma-Aldrich). Four $\mathrm{mL}$ of blood specimen were diluted with $2 \mathrm{~mL}$ of $1 \times$ PBS (in a total volume of $6 \mathrm{~mL}$ ) and were layered on the top of the ficoll layers. After a centrifugation step at room temperature for 30 minutes at 2,000rpm, the PMN were isolated from the interface between the two ficoll layers and washed with $1 \times$ PBS for 10 minutes at $1.600 \mathrm{rpm}$. Finally, cells were re-suspended in $1 \times$ PBS for subsequent immunophenotyping with the specific monoclonal antibodies, as described above. In case that erythrocytes were present, a lysis step with $\mathrm{NH}_{4} \mathrm{Cl} / \mathrm{KHCO}_{3}$, before staining procedure, was performed.

PMN culture assays: In a 24 -well tissue culture plate, $2 \times 10^{5} \mathrm{WBC}$ (containing at least 1-1.5 $\times 10^{5} \mathrm{PMN}$ ) were seeded in a total volume of 500 1L Iscoves Basal Medium (Biochrom, Berlin, Germany), containing $6 \%$ normal human serum (NHS) with or without the presence of LPS (100 ng/mL, E.Coli serotype 026:B6, Sigma-Aldrich). After 10 minutes of pre-incubation in order to let the cells rest in a humidified atmosphere $\left(37^{\circ} \mathrm{C}, 8 \% \mathrm{CO}_{2}\right)$, WBC were treated with LPS for $1 \mathrm{~h}$. Afterwards, cells were collected, washed, lysed in order to remove erythrocytes, stained with the specific antibodies and the proper isotype controls, and evaluated by flow cytometry, as described above.

\section{Statistical analysis}

Wilcoxon non-parametric match-paired analysis was performed for the comparison of different experimental conditions. Presented data refer to Intensity of expression defined by the formula: Intensity of expression $=\%$ of expression $\times$ MFI. Statistical analyses and graphs were made on GraphPad-6 Prism software (CA, USA). For all analyses, alpha was set at 0.05 (2-sided).

\section{Results}

\section{Expression of immunoglobulin receptors (CD16, CD64)}

The Fc receptor with low affinity for IgG (FCGR3 or CD16; OMIM $\left.{ }^{*} 610665\right)$ is expressed constitutively on mature PMN, and its crosslinking leads to degranulation and activation of the respiratory burst [9]. As presented in Figure 1A, CD16 was significantly upregulated after isolation, as well as after incubation of whole blood without any trigger ( $p=0.001$ and $p=0.002$, respectively). However, when PMN were treated with LPS, they exhibited a lower intensity of expression comparing to both untreated incubated PMN $(p=0.001)$ and PMN in resting (basal) state $(p=0.005)$. CD64 is a high affinity IgG receptor (OMIM $\left.{ }^{*} 601502\right)$ that is expressed only in activated granulocytes and is a useful marker in infectious conditions [10,11], and sepsis [12]. We did not find any remarkable CD64 expression in all experimental conditions, apart from two (2) individuals exhibiting over $20 \%$ expression in basal state ( $20.3 \%$ and $42.4 \%$, respectively), with its intensity of expression to being decreased during both incubation conditions (with and without LPS, Figure 1B).

\section{Expression of maturation markers (CD10)}

$\mathrm{CD} 10$ (OMIM $\left.{ }^{\star} 120520\right)$ is a specific marker that is indicative of maturation state of PMN [13]. We observed that the majority of PMN express CD10 in all cases, and the intensity of its expression is significantly increased after PMN isolation $(p<0.001)$ (Figure $1 C)$. On the other hand, no significant differences were observed considering the other experimental conditions (Figure 1C).

\section{Expression of adhesion molecules (CD11b, CD18, and CD66b)}

CD18 (OMIM ${ }^{*} 600065$ ) is the beta subunit of 12 -integrins. It forms heterodimers with four distinct alpha subunits (CD11a, CD11b, CD11c, CD11d), creating surface receptors that are involved in cell adhesion, but function also as complement receptors [14,15]. CD11b (OMIM $\left.{ }^{*} 120980\right)$, along with CD18 forms the macrophage-1 antigen (MAC-1), one of the most important structures mediating the arrest of neutrophils during their adhesion to the activated endothelium $[16,17]$. Moreover, the CD11b/CD18 complex represents the complement receptor 3 (CR3) that recognizes $\mathrm{iC} 3 \mathrm{~b}$ when bound to the surface of foreign cells, as well as several bacterial saccharides, like LPS [18].

We observed that the intensity of CD18 expression was higher after isolation $(p<0.001)$ and incubation of whole blood neutrophils, with ( $p<0.001)$ or without LPS $(p=0.002)$, comparing to resting cells. The difference of expression was not significant when the comparison was made between neutrophils under culture conditions $(p=0.090)$ (Figure 1D). Furthermore, a remarkable increase of the intensity of CD11b expression was also observed in all experimental conditions (Figure $1 \mathrm{E})$.

CD66b (OMIM: 615747) is a specific granulocytic marker that is upregulated after LPS activation, reflecting a degranulation phenomenon [19]. It belongs to Carcino-Embryonic Antigen Cell Adhesion Molecule (CEACAM) family and its stimulation induces oxidative burst and increases neutrophil adhesion via a CD18- dependent pathway [20]. We observed that CD66b expression was also significantly increased in all experimental conditions, compared to resting cells (Figure 1F). In addition, a remarkable increase of CD66b expression was found in LPStreated PMN compared to untreated ones in culture conditions $(p<$ 0.001 ), although a notable variation of CD66b expression was observed after LPS exposure (as presented in detail, in Figure 1F).

\section{Discussion and conclusion}

PMN are cells of the innate immune system comprising the first line host defense against invading microorganisms. Their life begins in the bone marrow, where progenitor cells of myeloid origin differentiate into mature PMN, entering the circulation as terminally differentiated 

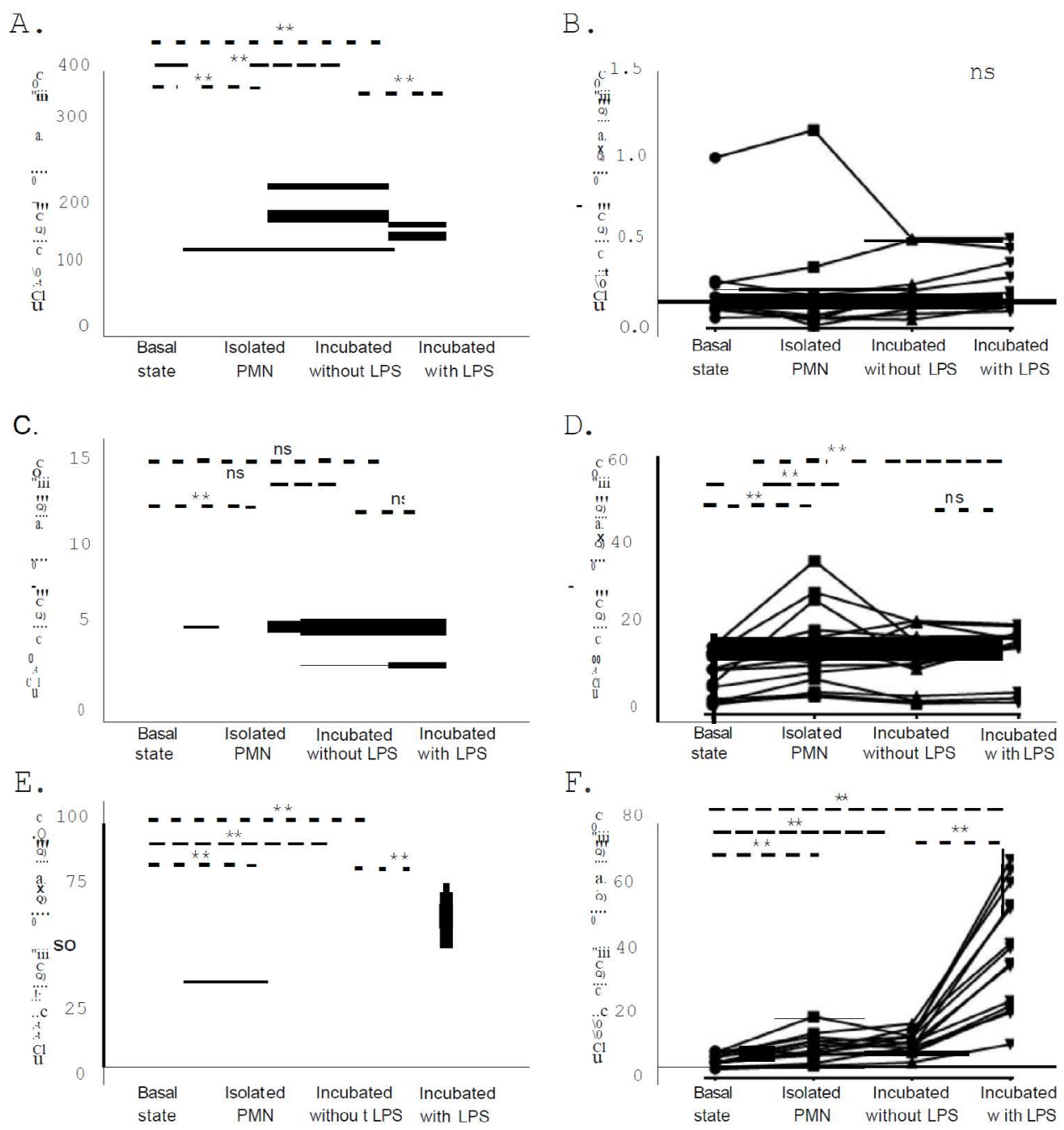

Figure 1. Immunophenotyping of polymorphonuclear cells. A. CD16 absolute intensity of expression. B. CD64 absolute intensity of expression. C. CD10 absolute intensity of expression. D. CD18 absolute intensity of expression. E. CD11b absolute intensity of expression. F. CD66b absolute intensity of expression. Basal State: Whole Blood neutrophils at resting state. Isolated PMN: Resting state neutrophils, after isolation procedure. Incubated without LPS: Whole blood Neutrophils in culture conditions without any trigger. Incubated with LPS: Whole Blood neutrophils in culture conditions treated with LPS. Intensity of expression $=(\%$ of expression $\mathrm{x}$ MFI $) / 100$. Each dot represents one subject; each subject's treatment condition is connected with lines. ${ }^{* *}: p<0.01 .{ }^{*}: p<0.5$. ns: $p>0.5$

cells. They die by apoptotic cell death, having a short lifespan of 6-12 hours in the periphery, unless they enter the tissue phase following an inflammatory stimulus, where they display a prolonged lifespan. After exposure to inflammatory stimuli, PMN can produce cytokines and chemokines, regulating inflammation $[21,22]$. There is increased evidence that changes in the expression of several molecules can be used as a quantifiable index of PMN activation, after treatment with different stimuli, or can be interpreted as a "primed" status of the cell [21]. PMN activation and degranulation result in the exposure of their contents in both the plasma membrane and the extracellular milieu; however, the level of expression of different PMN markers usually reaches a plateau and it is not altered by subsequent stimuli [23].

In our study, we investigated simultaneously the effect of common experimental conditions on healthy individuals' PMN. Our findings indicate that the expression of PMN surface molecules are affected by their experimental manipulation. In particular, we observed that CD10 and CD16 are more prone to unspecific priming due to isolation procedure, while the adhesion molecules $\mathrm{CD} 11 \mathrm{~b}$ and $\mathrm{CD} 66 \mathrm{~b}$ are more susceptible to culture conditions, even in the absence of any trigger. On the other hand, CD66b expression exhibits a great variation of its intensity in response to LPS, while CD64 is expressed in low levels on PMN and it is not affected by experimental manipulation, even by LPS exposure. These findings should be taken into consideration when studying PMN defects, since abnormalities of PMN compartment have been implicated in the pathogenesis and/or phenotype of several diseases, including sepsis [24] and immunodeficiencies [25]. PMN defects can affect the response to pathogens through several mechanisms, like phagocytosis, chemotaxis and transmigration [26], or disturb the dialectic communication of neutrophils with the other counterparts of the immune system [27]. There are several reports in the literature analyzing the effect of several experimental conditions on PMN functions, including isolation [28,29], the usage of different anticoagulants [8], the method of fixation or the effect of temperature alterations [21] on PMN immunophenotyping, or functional responses to $\mathrm{Ca}^{2+}$ homeostasis, ROS production, phagocytosis and/or bacterial ingestion [28]. However, the data from different studies cannot be compared and most importantly they lead to controversial and opposite 
findings. In our study, we focused on PMN immunophenotyping, as it is a fast procedure to detect possible defects, and for this purpose we analyzed common surface markers indicative of PMN functional capacity. According to the data presented herein, we recommend that a minimal experimental manipulation of PMN should be chosen when investigate their functional capacity, to both avoid the unspecific priming and to draw safer conclusions when interpreting in vivo phenomena.

\section{Acknowledgments}

We would like to thank the volunteers and Ms Ioanna Papamargariti (nurse of the Primary Immunodeficiency Outpatient Clinic of University Hospital of Larissa) for blood collection.

\section{References}

1. Borregaard N (2010) Neutrophils, from marrow to microbes. Immunity 33: 657-670. [Crossref]

2. Papayannopoulos V, Zychlinsky A (2009) NETs: a new strategy for using old weapons. Trends Immunol 30: 513-521. [Crossref]

3. von Bernuth $\mathrm{H}, \mathrm{Ku} \mathrm{CL}$, Rodriguez-Gallego C, Zhang S, Garty BZ, et al. (2006) A fast procedure for the detection of defects in Toll-like receptor signaling. Pediatrics 118: 2498-2503. [Crossref]

4. Bouma G, Doffinger R, Patel SY, Peskett E, Sinclair JC, et al. (2009) Impaired neutrophil migration and phagocytosis in IRAK-4 deficiency. Br J Haematol 147: 153156. [Crossref]

5. Elghetany ME (2002) Surface antigen changes during normal neutrophilic development: A critical review. Blood Cells Mol Dis 28: 260-274. [Crossref]

6. Kuijpers TW, Tool AT, van der Schoot CE, Ginsel LA, Onderwater JJ, et al. (1991) Membrane surface antigen expression on neutrophils: a reappraisal of the use of surface markers for neutrophil activation. Blood 78: 1105-1111. [Crossref]

7. Youssef PP, Mantzioris BX, Roberts-Thomson PJ, Ahern MJ, Smith MD (1995) Effects of ex vivo manipulation on the expression of cell adhesion molecules on neutrophils. $J$ Immunol Methods 186: 217-224. [Crossref]

8. Freitas M, Porto G, Lima JL, Fernandes E (2008) Isolation and activation of human neutrophils in vitro. The importance of the anticoagulant used during blood collection. Clin Biochem 41: 570-575. [Crossref]

9. Rollet-Labelle E, Vaillancourt M, Marois L, Newkirk MM, Poubelle PE, et al. (2013) Cross-linking of IgGs bound on circulating neutrophils leads to an activation of endothelial cells: possible role of rheumatoid factors in rheumatoid arthritis-associated vascular dysfunction. J Inflamm (Lond) 10: 27. [Crossref]

10. Li S, Huang X, Chen Z, Zhong H, Peng Q, et al. (2013) Neutrophil CD64 expression as a biomarker in the early diagnosis of bacterial infection: a meta-analysis. Int $J$ Infect Dis 17: e12-23. [Crossref]

11. Qian W, Huang GZ (2016) Neutrophil CD64 as a marker of bacterial infection in acute exacerbations of chronic obstructive pulmonary disease. Immunol Invest 45: 490-503. [Crossref]
12. Davis BH, Olsen SH, Ahmad E, Bigelow NC (2006) Neutrophil CD64 is an improved indicator of infection or sepsis in emergency department patients. Arch Pathol Lab Med 130: 654-661. [Crossref]

13. Orr Y, Taylor JM, Bannon PG, Geczy C, Kritharides L (2005) Circulating CD10-/ CD16low neutrophils provide a quantitative index of active bone marrow neutrophil release. Br J Haematol 131: 508-519. [Crossref]

14. Arnaout MA (1990) Structure and function of the leukocyte adhesion molecules CD11/ CD18. Blood 75: 1037-1050. [Crossref]

15. Wright SD, Weitz JI, Huang AJ, Levin SM, Silverstein SC, et al. (1988) Complement receptor type three (CD11b/CD18) of human polymorphonuclear leukocytes recognizes fibrinogen. Proc Natl Acad Sci U S A 85: 7734-7738. [Crossref]

16. Balsam LB, Liang TW, Parkos CA (1998) Functional mapping of CD11b/CD18 epitopes important in neutrophil-epithelial interactions: a central role of the I domain. $J$ Immunol 160: 5058-5065. [Crossref]

17. Tan SM (2012) The leucocyte $\beta 2$ (CD18) integrins: the structure, functional regulation and signalling properties. Biosci Rep 32: 241-269. [Crossref]

18. Ross GD, Vetvicka V (1993) CR3 (CD11b, CD18): a phagocyte and NK cell membrane receptor with multiple ligand specificities and functions. Clin Exp Immunol 92: 181184. [Crossref]

19. Schmidt T, Brodesser A, Schnitzler N, Gruger T, Brandenburg K, et al. (2015) CD66b Overexpression and loss of C5a receptors as surface markers for staphylococcus aureus-induced neutrophil dysfunction. PloS One 10: e0132703. [Crossref]

20. Ruchaud-Sparagano MH, Stocks SC, Turley H, Dransfield I (1997) Activation of neutrophil function via CD66: differential effects upon beta 2 integrin mediated adhesion. Br J Haematol 98: 612-620. [Crossref]

21. Németh T, Mócsai A (2016) Feedback Amplification of Neutrophil Function. Trends Immunol 37: 412-424. [Crossref]

22. Naranbhai V, Fairfax BP, Makino S, Humburg P, Wong D, et al. (2015) Genomic modulators of gene expression in human neutrophils. Nat Commun 6: 7545. [Crossref]

23. Macey MG, Jiang XP, Veys P, McCarthy D, Newland AC (1992) Expression of functional antigens on neutrophils. Effects of preparation. J Immunol Methods 149: 37-42. [Crossref]

24. Sônego F, Castanheira FV, Ferreira RG, Kanashiro A, Leite CA, et al. (2016) Paradoxical Roles of the Neutrophil in Sepsis: Protective and Deleterious. Front Immunol 7: 155. [Crossref]

25. Malech HL, Hickstein DD (2007) Genetics, biology and clinical management of myeloid cell primary immune deficiencies: chronic granulomatous disease and leukocyte adhesion deficiency. Curr Opin Hematol 14: 29-36. [Crossref]

26. Keszei M, Westerberg LS (2014) Congenital defects in neutrophil dynamics. J Immunol Res 2014: 303782. [Crossref]

27. Robb CT, Regan KH, Dorward DA, Rossi AG (2016) Key mechanisms governing resolution of lung inflammation. Semin Immunopathol 38: 425-448. [Crossref]

28. Macey MG, McCarthy DA, Vordermeier S, Newland AC, Brown KA (1995) Effects of cell purification methods on CD11b and L-selectin expression as well as the adherence and activation of leucocytes. J Immunol Methods 181: 211-219. [Crossref]

29. Mosca T, Forte WC (2016) Comparative Efficiency and Impact on the Activity of Blood Neutrophils Isolated by Percoll, Ficoll and Spontaneous Sedimentation Methods. Immunol Invest 45: 29-37. [Crossref]

Copyright: (C2018 Tsinti G. This is an open-access article distributed under the terms of the Creative Commons Attribution License, which permits unrestricted use, distribution, and reproduction in any medium, provided the original author and source are credited. 